\title{
ALGUNAS ASOCIACIONES VEGETALES DE LOS TERRENOS DEL LAGO DE TEXCOCO.
}

\author{
Por J. RZEDOWSKI (1) \\ de la S. B. M.
}

\section{I.-DATOS HISTORICO-GEOGRAFICOS}

El Lago de Texcoco ocupa la porción más baja de la cuenca hidrográfica conocida con el nombre de Válle de México. Está situado aproximadamente en el centro de la cuenca, limitando en su parte occidental con la ciudad de México.

Como es bien sabido el Valle de México careció durante épocas prolongadas de drenaje, constituyendo una cuenca de tipo endorreico con una considerable extensión lacustre en su fondo. Los sedimentos acarreados por las corrientes fluviales y procedentes de la erosión de rocas y suelos han ido rellenando a través de los tiempos el fondo hasta elevarlo al nivel actual.

En el siglo XV, a la llegada de los conquistadores europeos existía todavía una superficie lacustre de tamaño muy grande. En un mapa cuya confección se atribuye a Hernán Cortés es posible observar un enorme lago, reconociéndose una porción dulce y una porción salada.

El desarrollo de un gran centro de población pronto hizo sentir su influencia sobre el reservorio acuático. Esta influencia iba constantemen-

(1) Trabajo realizado en el año 1954. Dirección actual del autor: Laboratorio de Botánica, Universidad Autónoma de San Luis Potosi, San Luis Potosi, México.

Rzedowski J. 1957. Algunas asociaciones vegetales de los terrenos del lago de Texcoco. Boletín de la Sociedad Botánica de México 21: 19-33. 


$$
\text { Venta de Carpio Totolcingo }
$$

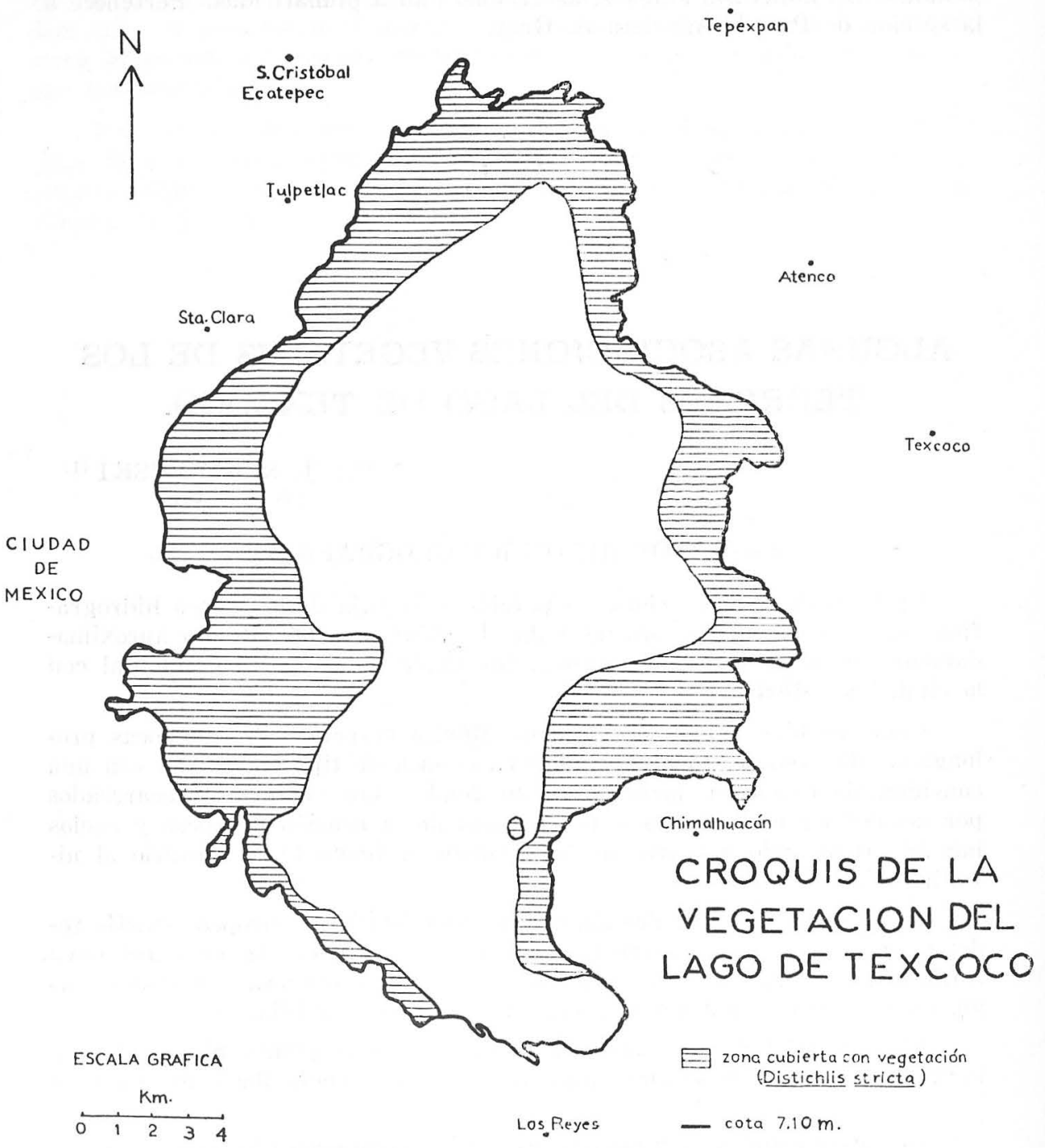


te en aumento hasta que las necesidades de la ciudad hicieron que a fines del siglo pasado se abriera artificialmente la cuenca, lo que redujo la dimensión de la superficie lacustre a menos de la mitad. Hoy día existen sólo restos del antiguo lago, representados por tres unidades completamente separadas entre sí y todas ellas en vías de desaparición. Estas son: 10. el Lago de Xochimilco, convertido en realidad en un grupo de canales alimentados por manantiales; 2o. el Lago de Texcoco, situado en la parte oriental de lo que era antiguamente el gran Lago de Tenoxtitlán y 3o. el Lago de Zumpango, de pequeña extensión.

El Lago de Texcoco carece de límites fijos. En el año de 1521, cuando constituía sólo una parte de la extensión lacustre continua, sus riberas llegaban por el occidente hasta los poblados de Tlalnepantla, Atzcapotzalco, Tacuba, Tacubaya y Mixcoac y sus aguas bañaban todo lo que es hoy el centro de la ciudad de México, alcanzando una superficie de más de 700 $\mathrm{km}^{2}$. (Flores, 1918). En la actualidad, en la época seca el Lago se reduce a unos cuantos charcos y en tiempo de aguas, habiendo precipitación abundante, puede alcanzar una superficie hasta de unos $100 \mathrm{~km}^{2}$. Modernamente se suele emplear la llamada cota de los $7.10 \mathrm{~m}$. sobre el plano de comparación del Valle, correspondiente a la altura aproximada de $2240 \mathrm{~m}$. sobre el nivel del mar, como un límite convencional del Lago, encerrando en tal forma unos $270 \mathrm{~km}^{2}$. Por razones de conveniencia se admitirá también este límite en el presente trabajo. Por un punto de situación casi central de esta área pasan el meridiano $99^{\circ}$ de longitud oeste y el paralelo $19^{\circ} 30^{\prime}$ de latitud norte.

Las porciones sur y poniente del área han sido objeto de obras destinadas a la bonificación de tierras. Toda esta zona está atravesada por multitud de canales, zanjas, caminos, que han servido para tal objetivo. Los canales más importantes son: El Gran Canal del Desagüe, el Canal de Sales, el Canal de Ayotla, el Canal de los Ríos de Churubusco y de la Piedad. El agua de la mayor parte de estos vasos confluye a una instalación especial destinada a la concentración de sales para su utilización industrial y que tiene la forma de un caracol. Gracias a las citadas obras de bonificación, realizadas principalmente por el método de lavado, una zona de aproximadamente $25 \mathrm{~km}^{2}$. se destina actualmente a la agricultura. Por otra parte y como resultado del impacto de la ciudad, grandes áreas están siendo urbanizadas, o bien ocupadas por industrias, aeropuertos, vías de comunicación, etc.

\section{II.-CONDICIONES ECOLOGICAS GENERALES}

El Lago de Texcoco es de naturaleza salobre y alcalina. El fenómeno de acumulación de sales en el fondo de cuencas hidrográficas cerradas puede explicarse fácilmente en términos de evaporación y concentración de estas substancias minerales procedentes de la erosión de materiales sóli- 
dos de las partes superiores de la cuenca. En el continente americano y en otras partes del mundo hay muchos lagos actuales y antiguos de naturaleza salina que revisten este origen. Los alcalinos son más escasos y su formación es más difícil de explicarse. Se supone que el carbonato de sodio, a cuya presencia se debe la concentración elevada de hidroxiliones, procede de la descomposición de rocas ricas en sodio, en este caso de las andesitas de hornblenda, rocas ígneas muy abundantes en la región, en que predominan los feldespatos. Es posible que el sodio sea transportado en forma de bicarbonato y más tarde por pérdida de anhidrido carbónico se convierta en carbonato (Orozco y Medinaveitia, 1941).

Es imposible hablar de una concentración determinada de sales a hidroxiliones del agua del Lago de Texcoco. Esta sufre cambios constantes y muy acentuados por efecto de las estaciones y de otros factores. A continuación se resumen datos de análisis de tres muestras tomadas en el mismo sitio (Curva de Patito) en diferentes épocas del año (proporcionados gentilmente por la Comisión Hidrológica del Valle de México):

Fecha

Mayo 14, 1953

Nov. 27, 1953

Enero 13, 1954

\begin{abstract}
$\mathrm{Cl}$
\end{abstract}
n.d

2.1

8.9

$\mathrm{Na}$

$\mathrm{HCO}_{2}$

$\mathrm{CO}_{3}$

$\mathrm{SO}_{4}$

$\mathrm{pH}$

20.7

25.8

2.1

1.9

8.9

1.7

9.6

Nota: las cantidades de sales se señalan en partes por mil.

Un fenómeno de suma importancia tanto para la fisonomía del Lago como para la flora y fauna que lo habita es la extrema inestabilidad del nivel lacustre. El Lago de Texcoco en los tiempos históricos probablemente nunca fue muy profundo. Hoy día, en tiempo de lluvias, rara vez llega a $0.5 \mathrm{~m}$. en los lugares más hondos. Es fácil comprender que este escaso volumen de agua se evapora rápidamente al agotarse las fuentes de alimentación en los primeros meses del año. El Lago se seca casi por completo, dejando al descubierto su fondo para volver a cubrirse con una capa de agua cuya extensión y profundidaci dependen en cierto grado del volumen de la precipitación, pero más bien del hombre, quien a su voluntad regula el escurrimiento en la cuenca mediante un sistema de presas, canales, desviaciones y túneles.

El clima de la región del Lago corresponde en términos generales al de la parte baja del Valle de México, pudiendo aplicársele la fórmula Cwbg del sistema de Koeppen. Sus características más sobresalientes son: temperatura anual cerca de $14^{\circ} \mathrm{C}$, pequeñas fluctuaciones anuales y fuertes fluctuaciones diurnas de la temperatura, aunque esta rara vez baja de los $0{ }^{\circ} \mathrm{C}$; precipitación anual de $600 \mathrm{~mm}$., cuyo $90 \%$ corresponde a los meses de mayo a octubre; evaporación anual de $2000 \mathrm{~mm}$; presión atrnosférica de $580 \mathrm{~mm}$.; iluminación fuerte; humedad atmosférica media de $60 \%$. 
El fondo del Lago sirve de sostén y hace las veces del suelo para las plantas acuáticas arraigadas así como para las plantas terrestres que se desarrollan en terrenos denudados. Como todo fondo lacustre el del Lago de Texcoco está formado por material sedimentario aluvial. Pero por falta de drenaje y debido a la evaporación fuerte se depositaron no solamente materiales en suspensión, sino también las sales solubles, formando una capa impregnada con estas últimas de muchos metros de profundidad.

Los suelos salobres alcalinos poseen características especiales y se agrupan dentro de la categoría de solonetz, llamada también álcali negro o suelo tequesquitoso (González Gallardo, 1941). Un rasgo característico de esta clase de suelos consiste en presentar movimientos verticales de sales que son cíclicos en escala anual. Este fenómeno es particularmente acentuado en regiones áridas y semiáridas. Durante la época seca las sales son arrastradas hacia la superficie por el agua que se evapora y a menudo llegan a formar una costra blanca de material cristalizado; en la época de lluvias en cambio estas sales se sumergen temporalmente a niveles más profundos. Los suelos alcalinos son arcillosos, casi amorfos e impermeables, a menudo forman una masa gelatinosa que al secarse se agrieta y forma figuras características. En el caso del Lago de Texcoco la capa arcillosa forma el horizonte $B$ de espesor muy considerable, por encima de él existe algunas veces un horizonte $\mathrm{A}$ de pocos centímetros de grosor, constituído por migajón arcilloso. La aita concentración de hidroxiliones impide casi por completo la vida de microorganismos del suelo y la cantidad de humus es insignificante.

La composición cuantitativa de sales solubles en los suelos del Lago de Texcoco presenta variaciones no solamente en dependencia de la estación del año sino también en los sentidos horizontal y vertical. Desgraciadamente no ha sido posible obtener datos fidedignos de análisis químicos de suelos de la región. En la literatura se admite un valor de $4 \%$ de sales solubles totales como promedio. No obstante la falta de datos cuantitativos, basándose en la distribución de la vegetación, se puede deducir que la salinidad aumenta por regla general yendo de la periferia hacia el centro del Lago.

Tratando de interpretar algunos análisis en lo referente a la proporción de las sales se puede deducir que del total de sales solubles el sodio constituye aproximadamente el $90 \%$ de los cationes, mientras que los cloruros y los carbonatos $(47 \%$ y $45 \%)$ forman la mayor parte de los aniories. Esta elevada cantidad de carbonatos es causante de que el $\mathrm{pH}$ alcance valores hasta de 10 y 11.

Otro factor ambiental de gran trascendencia lo constituye indudablemente el hombre. Desde tiempos precortesianos sus actividades se traducían en el azolve y la desecación del Lago. Con el fin de utilizar las tierras ganadas al Lago para la agricultura se ha practicado lavados por percolación eliminando así una gran cantidad de sales. Desde 1911 se está tra- 
tando de recubrir los terrenos desecados con un manto de vegetación. Para conseguir este objetivo se han indicado obras en gran escala, particularmente en las porciones sur y oeste de la región en estudio. Para facilitar los lavados se ha establecido una red de canales y caminos. Se han introducido especies arbóreas exóticas iniciando la aforestación, se han propagado artificialmente las especies nativas (Quevedo, 1922), (González, 1933). Actualmente las obras están prácticamente suspendidas por falta de agua suficiente para la bonificación de las tierras.

De cierta importancia como factor ecológico debe considerarse asímismo el pastoreo, que en algunas regiones del Lago es bastante intenso.

En resumen fácilmente se desprende que el Lago de Texcoco, tal como se encuentra en la actualidad, ofrece condiciones muy difíciles para el desarrollo de especies vegetales. Son dos los principales factores de adversidad: 1. la salinidad y la alcalinidad del agua y del suelo, y 2. la inestabilidad del nivel del agua. Los organismos que se aventuran a ocupar este hábitat están obligados a presentar adaptaciones específicas al respecto.

\section{III.-CARACTERISTICAS GENERALES DE LA VEGETACION}

Este trabajo se ocupará exciusivamente de la vegetación macroscópica y siempre que se emplee la palabra vegetación debe entenderse en esta acepción restringida.

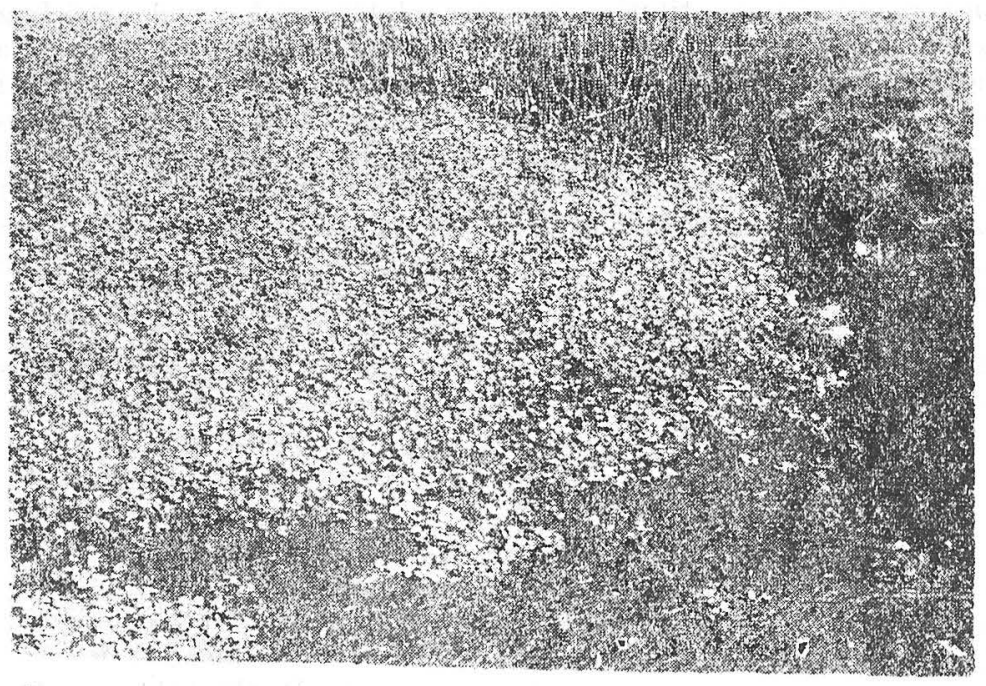

Fig. 1. Eichornia crassipes y Scirpus pungens en una zania. 
Como consecuencia de las condiciones extremosas sus rasgos principales consisten en la escasez de especies, escasez de individuos, escasez de formas conspicuas, falta casi completa de estratificación, y por lo tanto un monto y volumen limitado de materia orgánica vegetal. La mayor parte de la superficie comprendida dentro de la cota 7.10 es desde el punto de vista adoptado completamente estéril. Es la zona de la mayor concentración salina y la afectada por desecaciones e inundaciones periódicas (véase mapa adjunto).

Las plantas adaptadas a desarrollarse en regiones salobres forman el tipo ecológico llamado halophytia. Un gran número de halófitas presentan estructura suculenta de sus órganos vegetativos, otras como p.e. Distichlis spicata eliminan sales mediante el proceso conocido como gutación. Como regla general las halófitas reducen considerablemente su desarrollo en la época seca que corresponde a la mayor concentración salina en la capa superficial del suelo.

No todas las especies vegetales que habitan los terrenos del Lago de Texcoco se clasifican dentro del grupo de las halófitas. Aunque mucho menos abundantes, existe un gran número de especies que se desarrollan también en condiciones diferentes. Pertenecen aquí los vegetales que viven en las orillas de las zanjas y en éstas, cerca de caminos, campos cultivados y pastoreados y en algunos otros sitios. Una gran parte de estas plantas está constituída por especies ruderales, que, como es sabido, poseen adaptaciones para desarrollarse en habitats antropógenos que generalmente también son abundantes en sales.

Mientras que a un grupo de vegetales debe considerarse como hidrófitos (las plantas acuáticas, las de las zanjas, etc.), otros poseen características de xerófitos.

Aunque no es posible establecer un límite preciso, para describir la vegetación de los terrenos del Lago de Texcoco conviene dividirla en acuática $y$ terrestre.

\section{IV.-VEGETACION ACUATICA}

Es muy probable que hace todavía unos 150 años el Lago de Texcoco haya tenido una vegetación acuática abundante. En la actualidad su desarrollo está limitado por falta de habitats adecuados. Los únicos sitios que sostienen vegetación acuática fanerogámica son: 1. algunos canales, zanjas y desembocaduras de arroyos, y 2. lugares cercanos a manantiales y pequeños contingentes lacustres que conservan agua durante todo el año. 


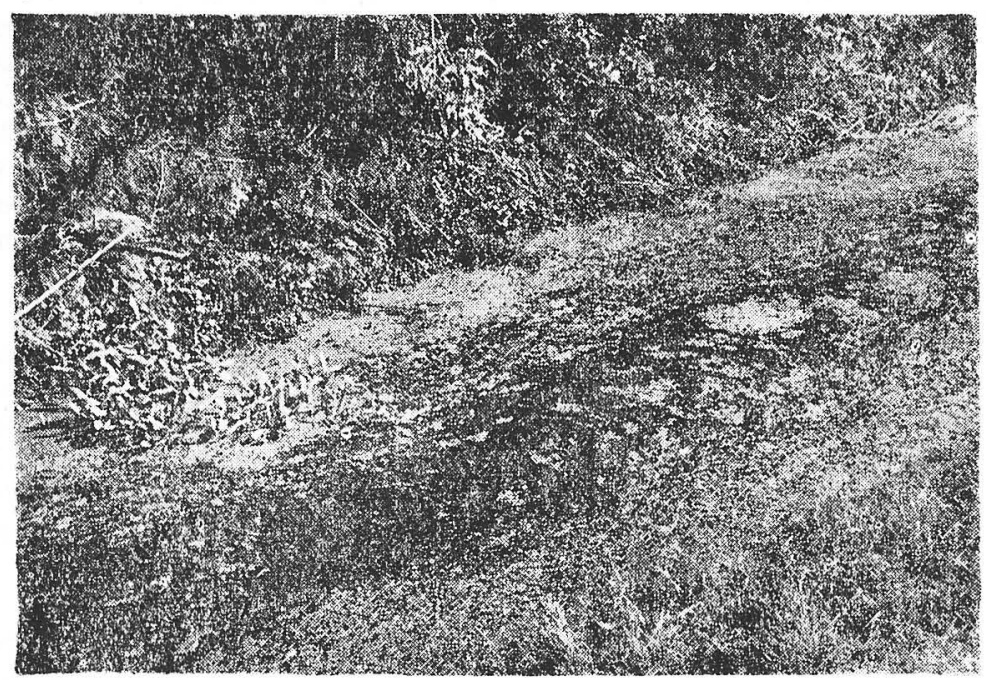

Lem na gibba y Polygonum punctatum en una zanja.

Tanto los canales y zanjas en cuestión, como las desembocaduras de arroyos se localizan en la periferie del terreno del estudio. Son habitats restringidos, con afluencia más o menos constante de agua dulce o poco salobre. La lista de especies que sigue comprende todas las observadas en el área. La terminología ecológica empleada en este lugar y más adelante es la propuesta por Huguet del Villar (1929).

\section{Helostadion:}

Aganipea bellidiflora

Cyperus Bourgaei

Eleocharis Dombeyana

Hydrocotyle verticillata

Juncus balticus

Iussieua repens

Leersia hexandra

Polygonum hydropiperoides

Polygonum punctatum

Sagittaria macrophylla

Scirpus lacustris

Scirpus pungens

Typha latifolia
Ploadostadion:

Nymphaea sp.

Potamogeton pectinatus

Baphostadion:

Myriophyllum hippuroides 


\section{Epipleon:}

Azolla caroliniana

Eichhornia crassipes

Lemma minor

Lemma gibba

Lemma valdiviana

Wolffia columbiana
Hypopleon:

Ceratophyllum demersum

Es preciso señalar que en ninguna parte se han encontrado todas estas especies juntas y muchas veces sólo una o dos. Con excepción tal vez de Juncus balticus todas las plantas mencionadas son características de la vegetación de las aguas dulces del Valle de México (Reiche, 1923). Desde este punto de vista las comunidades presentes no serían sino asociaciones fragmentarias, seriales, perturbadas o restringidas y correspondientes a la asociación dulceacuícola típica, cuya parte forman además muchos otros elementos.

En la porción norte del Lago se encuentran varios manantiales de agua dulce; su presencia permite que existan todavía terrenos que no se desecan por completo en la primavera. La vegetación típica de estos

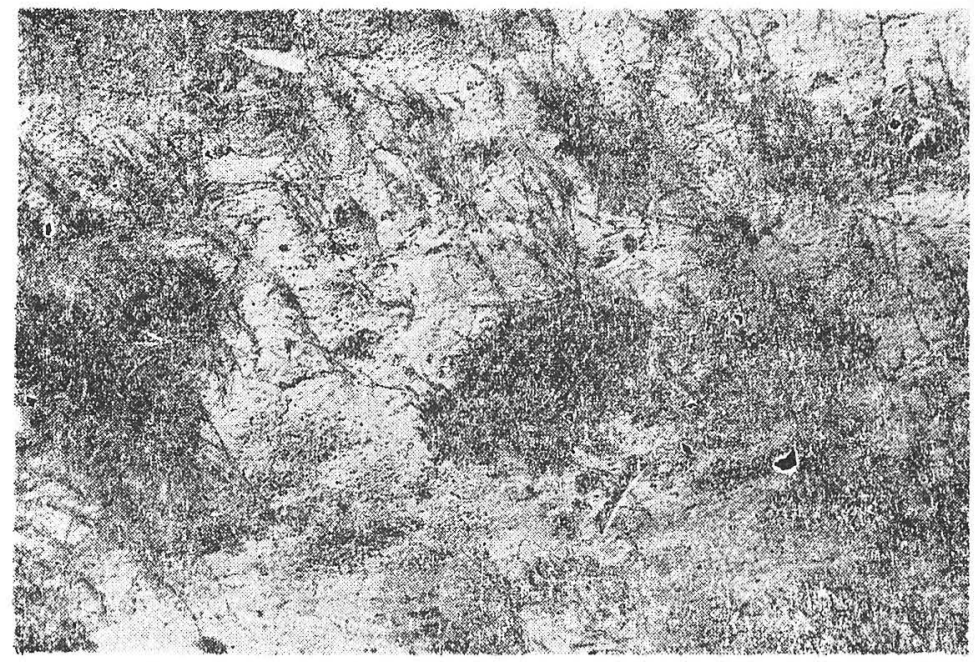

Manchón de Suaedanigra.

charcos consiste del tulillar de Juncus balticus, que ocupa sus orillas e islotes poco elevados y a menudo también fondos de escasa profundidad. 
Pocas son las especies que conviven en este medio con el tulillo. Pueden citarse por ejemplo Aster pauciflorus, Bacopa Monnieri, Scirpus pungens. En la proximidad inmediata de los manantiales y en un radio de varios metros se desarrollan manchones muy densos de Typha latifolia, especie robusta de 2 a 3 metros de altura. Acompañando a Typha puede encontrarse Scirpus pungens. Esta comunidad es indudablemente la más conspicua de todas las que se encuentran espontáneamente en el Lago de Texcoco.

\section{V.-VEGETACION TERRESTRE}

A medida que el Lago ha ido reduciéndose y ha ido disminuyendo el volumen del manto vegetal acuático, la vegetación terrestre gana cada vez más terreno. Es obvio que la vegetación de tierra firme que ocupa la superficie de un lago en vías de desaparición está aún muy relacionada con el agua. Muchos de sus componentes serán las mismas especies hidrófitas antes citadas; ello puede explicarse más o menos satisfactoriamente en términos de una amplia escala de adaptación, pero a la vez comprueba el hecho de que no es posible delimitar con precisión lo acuático de lo terrestre en este caso.

Conviene dividir la vegetación de tierra firme en: 1 vegetación de las llanuras del antiguo fondo, y 2. vegetación de las orillas de canales, zanjas, etc.

La comunidad típica de los llanos aluviales salobres es el zacahuistal o consocies de Distichlis spicata, que podría denominarse también estepa o pradera salina. Es la asociación vegetal más extendida del área en estudio. Rodea en forma de un anillo a la porción central desértica del Lago y sólo es interrumpida por caminos y los escasos reservorios más o menos permanentes de agua. La comunidad es sumamente agresiva y es la primera colonizadora (pioneer) de los terrenos emergidos. Su avance hacia el centro del Lago está limitado generalmente por la alcalinidad elerada que impide por completo su desarrollo; otras veces, sin embargo, como puede comprobarse, es la inundación periódica la que ejerce el mismo efecto. Aunque teóricamente podrían distinguirse dos estratos: herbáceo y rasante, no parece conveniente establecer esta división, puesto que la misma especie frecuentemente se encuentra en uno u otro estrato. La gran agresividad y la resistencia de la comunidad a condiciones adversas se debe primordialmente a la especie dominante Distichlis spicata. Además de ella existen otros elementos florísticos mucho menos abundantes, en su mayor parte halófitos. El siguiente cuadro resume algunas de las características ecológicas de las especies constitutivas principales. 


\begin{tabular}{|c|c|c|c|c|c|}
\hline Especie & biotipo & $\begin{array}{c}\text { categoría } \\
\text { fisonómica } \\
\text { de } \\
\text { Raunkiaer }\end{array}$ & $\begin{array}{c}\text { grado de } \\
\text { constancia } \\
\text { social }\end{array}$ & $\begin{array}{l}\text { grado de } \\
\text { expansión } \\
\text { horizontal }\end{array}$ & $\begin{array}{l}\text { socia- } \\
\text { bili- } \\
\text { dad }\end{array}$ \\
\hline Distichlis spicata & graminoidetum & $\mathrm{Ch}$ & 5 & 4 & cs \\
\hline Suaeda nigra & crassicaultetum & $\mathrm{Ch}$ & 3 & 1 & $\mathrm{~cm}$ \\
\hline Juncus balticus & graminoidetum & $\mathrm{Ch}$ & 2 & 1 & $\mathrm{gr}$ \\
\hline Chenopodium mexicanum & crassicauletum & $\mathrm{H}$ & 2 & 1 & sp \\
\hline Trianthema portulacastrum & crassicauletum & $\mathrm{H}$ & 2 & 1 & sp \\
\hline Sesuvium portulacastrum & crassicauletum & $\mathrm{H}$ & 2 & 1 & sp \\
\hline Atriplex muricata & crassicauletum & $\mathrm{H}$ & 2 & 1 & $\mathrm{sp}$ \\
\hline Heliotropium curassavicum & srassicauletum & $\mathrm{H}$ & 2 & 1 & $\mathrm{sp}$ \\
\hline Sporobolus argutus & graminoidetum & $\mathrm{Ch}$ & 2 & 1 & $\mathrm{~cm}$ \\
\hline Hordeum jubatum & graminoidetum & $\mathrm{H}$ & 2 & 1 & $\mathrm{~cm}$ \\
\hline Xanthocephalum humile & herbetum & $\mathrm{H}$ & 2 & 1 & sp \\
\hline
\end{tabular}

Para facilitar la descripción de la comunidad conviene distinguir en ella dos facies fundamentales. La primera se caracteriza por estar constituída exclusivamente de Distichlis spicata (zacahuistle) y Suaeda nigra (romerillo), la segunda admite la compañía de otras especies. Es difícil marcar un límite preciso entre estas facies, pero como regla general puede admitirse que la primera está situada más centralmente y la segunda más periféricamente con respecto al centro del Lago.

Tanto por su composición como por su situación, fácilmente se reconoce que la facies primera ocupa un habitat más extremo y desde el punto de vista sucesional es más primitiva. El zacahuistle tiene una expansión horizontal (grado de cobertura) media de un $70 \%$ y ejerce una dominancia absoluta. Sólo de cuando en cuando aparecen pequeños manchones de una o varias matas de romerillo, a menudo aislados mediante un espacio desnudo de la masa de la gramínea. Este hecho hace pensar en un antagonismo acentuado entre las dos especies. Distichlis, por estar probablemente mejor adaptada, gana más terreno, pero no lo suficiente para eliminar por completo a la quenopodiácea. El límite entre el zacahuistal y el área deshabitada es por lo general muy brusco, casi no habiendo disminución de extensión horizontal en la zona limítrofe. Es común, observar en estos sitios la tendencia colonizadora de individuos de Distichlis, que se extienden mediante sus rizomas en líneas más o menos rectas hacia el terreno desierto.

La facies segunda se desarrolla sobre suelos más tolerantes. Hacen su aparición aquí pocas especies, generalmente también halófitas, pero de menor grado de resistencia. Como puede notarse en el cuadro las más frecuentes pertenecen a los biotipos crassicauletum y graminoidetum, no 
existiendo ninguna planta leñosa. Con excepción de Suaeda nigra ninguna de las especies subordinadas tiene constancia social superior a 2, lo que significa que estas formas sólo se encuentran en determinadas porciones de la asociación y faltan por completo en otras. Este es el caso p.e. de la hidrófita Juncus balticus, que busca sitios de humedad más constante o de Hordeum jubatum, que sólo es abundante en la parte occidental. La expansión horizontal de Distichlis spicata es aproximadamente igual que en la facies anterior, pero la diferencia consiste en que es menor la cantidad y la superficie de manchones desnudos, siendo estos parcialmente cubiertos por las especies subordinadas.

Además de los elementos florísticos citados en el cuadro se han observado los siguientes como constitutivos de la comunidad, pero con constancia social igual a 1:

Ambrosia peruviana

Arenaria Bourgaei

Atriplex linifolia

Bouteloua simplex

Cyperus esculentus

Cyperus melanostachys

Euphorbia sp.

Euphrosyne parthenifolia

Leptochloa dubia

Lippia nodiflora

Lythrum alatum
Medicago denticulata

Muhlenbergia tenuiflora

Nierembergia angustifolia

Petunia parviflora

Ranunculus Donianus

Sanvitalia procumbens

Silvia serpyllifolia

Sonchus asper

Trifolium amabile

Xanthocephalum centauroides

En la vegetación de las orillas de canales, zanjas y otros pequeños depósitos de agua no puede distinguirse una comunidad vegetal definida. El área es demasiado chica y el hábitat influido constantemente por actividades humanas. Existen algunas especies típicas y probablemente de dispersión natural, otras sin embargo no son sino simples malezas. No será de extrañarse tampoco que se sitúen aquí algunos elementos ya citados como constituyentes de la vegetación acuática o de llanura.

Son propias de esta vegetación de los borces dos especies leñosas, poro cuya espontaneidad puede ponerse en duda. El ahuejote Salix Bonplandiana se encuentra en las proximidades de poblados, principalmente del lado oriente del Lago. El arbusto Baccharis glutinosa, en cambio, es frecuente del lado poniente, en la región donde más influencia ejercieron las "Obras del Lago de Texcoco" sobre las condiciones generales y sobre la vegetación. Además se pudieron observar como propios del habitat las siguientes especies: 
Agrostis semiverticillata

Ambrosia peruviana

Aster exilis

Atriplex muricata

Bacopa Monnieri

Beta vulgaris

Bidens chrysanthemoides

Cyperus Bourgaei

Cyperus esculentus

Cyperus melanostachys

Distichlis spicata

Echinochloa crusgalli

Echinochloa crusgalli var. zelayensis Eleocharis Dombeyana

Erigeron bonariensis
Erigeron scaposus

Juncus balticus

Medicago denticulata

Nasturtium mexicanum

Panicum repens

Polygonum hydropiperoides

Polygonum punctatum

Polypogon elongatus

Polypogon monspeliensis

Ranunculus cymbalaria

Ranunculus dichotomus

Rumex maritimus

Rumex mexicanus

Sagittaria macrophylla

Urtica dioica var. angustifolia

Esta vegetación herbácea no cubre por completo el suelo ni siquiera en la época lluviosa y su densidad y riqueza florística van disminuyendo a medida que se acerca hacia la parte central del Lago, desapareciendo más o menos al mismo nivel donde termina Distichlis spicata en terrenos planos.

\section{VI.-VEGETACION ANTROPOGENA}

En un lugar donde las condiciones del medio han sido modificadas en forma tan intensa es difícil muchas veces distinguir lo nativo y lo espontáneo de lo introducido y lo artificial. Como pudo notarse en los párrafos anteriores un buen número de especies que al parecer se han acomodado en las comunidades características, suelen considerarse como malezas en otros sitios. También se expresaron dudas acerca del origen de dos especies leñosas.

Aparte de estos elementos existen otros cuya distribución en el terreno claramente comprueba su procedencia. Se pueden dividir en dos grupos: 1. especies arbóreas introducidas, y 2. especies ruderales.

Entre las primeras las más frecuentes son:
Alnus acuminata
Populus mexicana
Buddleia cordata
Populus alba
Casuarina equisetifolia
Schinus molle
Eucalyptus spp.
Tamarix juniperina 
De las segundas pueden enumerarse:

Amaranthus hybridus

Argemone mexicana

Bidens pilosa

Brassica campestris

Buddleia sessiliflora

Chenopodium album

Chenopodium ambrosioides

Chloris virgata

Conyza sophiaefolia

Cynodon dactylon

Datura stramonium

Eleusine indica

Encelia mexicana

Eragrostis limbata

Erodium cicutarium

Eruca sativa
Galinsoga parviflora

Lepidium intermedium

Malva parviflora

Nicotiana glauca

Physalis aequata

Raphanus raphanistrum

Reseda luteola

Setaria geniculata

Sisymbrium irio

Solanum rostratum

Sphaeralcea angustifolia

Sporobolus atrovirens

Sporobolus Poiretii

Taraxacum officinale

Verbena carolina

y otras más.

Las actividades de aforestación se concentraron en la región próxima al Peñón de los Baños y San Juan de Aragón, en el borde occidental del Lago. Todas las especies citadas pueden encontrarse a lo largo de los caminos y canales más grandes, pero además Casuarina equisetifolia y

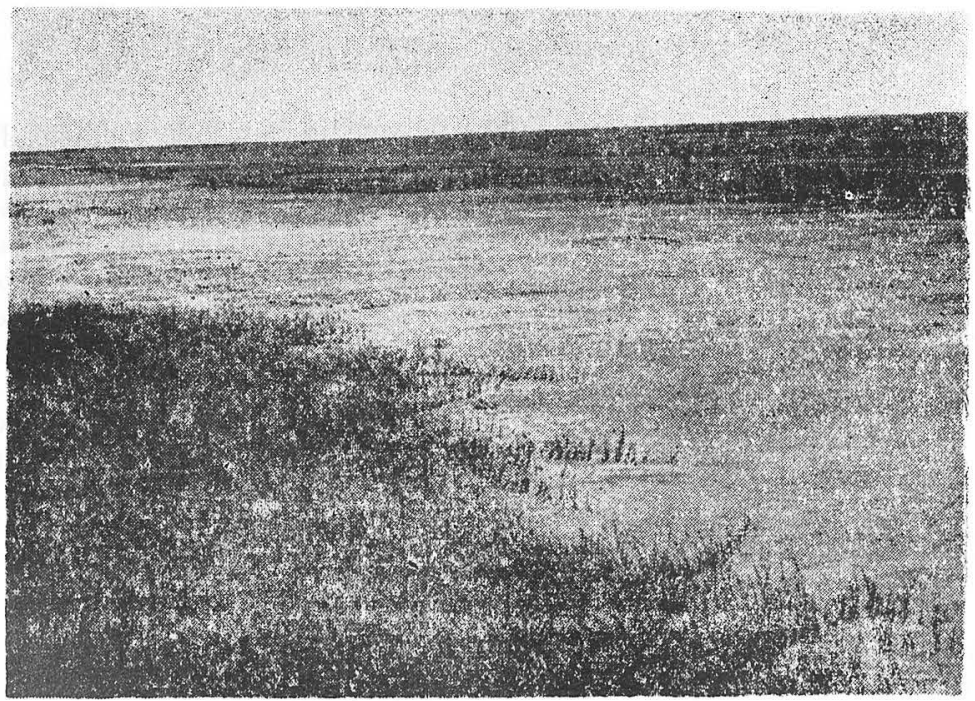

Limite entre la vegetación (de $D$ istich lis spicata) y de la región deshabitada. 
Eucalyptus spp. fueron plantados en masa sobre suelos lavados. La primera especie prosperó bastante bien y actualmente forma un pequeño bosque en una parte de estos terrenos.

Según datos de la literatura se han verificado también trabajos de propagación artificial de algunas especies nativas. Hoy sería difícil reconocer estos plantíos.

\section{LITERATURA CITADA}

Flores, T., 1918. El tequesquite del Lago de Texcoco. An. Inst. Geol. Méx. V.

González, G., 1933. El problema agrícola en los terrenos del antiguo vaso del Lago de Texcoco. Méx. Forest. XI : 133-142.

González Gallardo, A., 1941. Introducción al estudio de los suelos. Banco Nacional de Crédito Agrícola. México, D. F., 484 pp.

Huguet del Villar, E., 1929. Geobotánica. Editorial Labor. Barcelona, $339 \mathrm{pp}$.

Orozco, F. y Medinaveitia, A., 1941. Estudio químico de los lagos alcalinos. El origen del carbonato sódico. An. Inst. Biol. Méx. XII: 429438.

Quevedo, M. A., 1922. Las polvaredas de los terrenos tequezquitosos del antiguo Lago de Texcoco y los procedimientos de enyerbe para remediarlas. Mem. Soc. Cient. A. Alzate XL: 533.548.

Reiche, C., 1914. La vegetación en los alrededores de la capital de México. México, D. F., 143 pp. 